УДК 519.688:622.276

\title{
ДИАГНОСТИЧЕСКИЕ КРИТЕРИИ ВЫДЕЛЕНИЯ ФИЛЬТРАЦИОННЫХ ПОТОКОВ В ПРОЦЕССЕ ГИДРОДИНАМИЧЕСКИХ ИССЛЕДОВАНИЙ ГОРИЗОНТАЛЬНЫХ СКВАЖИН
}

\author{
Сергеев Виктор Леонидович1, \\ SVL00@tpu.ru
}

Донг Ван Хоанг' hoang.tpu@gmail.com

Хагай Данил Эдуардович², hagayd@mail.ru

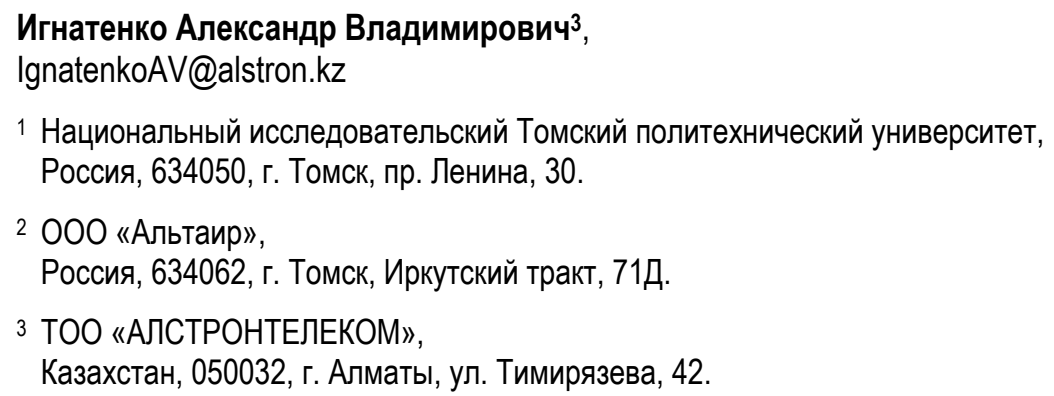

Актуальность работы обусловлена необходимостью выделения фильтрационных режимов течения для определения гидродинамических параметров нефтяных пластов в процессе проведения нестационарных испытаний горизонтальных скважин по кривым восстановления забойного давления.

Целью исследования является разработка диагностических критериев детерминированных моментов давлений для выделения фильтрационных режимов течения в процессе нестационарных гидродинамических испытаний горизонтальных скважин нефртяных месторождений по кривым восстановления давления.

Методы исследования основаны на теории и практике нестационарных испытаний горизонтальных скважин, линейной алгебре, идентификации систем, системном анализе. Апробация предложенных диагностических критериев осуществлялась $c$ использованием промысловых данных нестационарных испытаний нефтяных горизонтальных скважин по кривым восстановления забойного давления.

Результаты. Разработаны диагностические критерии выделения на кривой восстановления забойного давления участков раннего радиального, линейного и позднего радиального (псевдорадиального) потоков в процессе проведения гидродинамических исследований горизонтальных скважин нефтяных месторождений. В основе диагностических критериев использованы оценки детерминированных моментов давлений, полученные в процессе проведения испытаний горизонтальных скважин. Для определения неизвестных значений пластовых и забойных давлений на недовосстановленном участке кривой восстановления забойного давления использованы феноменологические прогнозирующие модели забойного давления с параметрами, зависящими от времени, с учетом экспертной оценки пластового давления, и адаптивные алгоритмы идентифрикации. Проводилась обработка результатов испытаний двух горизонтальных скважин нефтяного месторождения. Показано, что предложенные диагностические критерии практически не уступают по точности графооаналитическому методу выделения режимов фильтрации и позволяют определять время начала и завершения фильтрационных потоков, а также время завершения испытаний скважин в процессе их проведения без участия квалифицированного интерпретатора.

\section{Ключевые слова:}

Диагностические критерии, фильтрационные потоки, адаптация, горизонтальные скважины, гидродинамические исследования скважин, идентификация, кривые восстановления давления, нефтяные пласты.

\section{Введение}

Актуальной проблемой при обработке результатов нестационарных гидродинамических исследований (ГДИ) горизонтальных скважин является задача выделения на кривой восстановления давления (КВД) участков раннего радиального, линейного и позднего радиального потоков. В настоящее время основными методами выделения фильтрационных потоков являются аналитический и графоаналитический, основанный на визуальном анализе производной забойного давления (рис. 1) [1-10].

Однако в промысловых условиях использование этих методов в процессе проведения гидродинамиче- ских исследований скважин вызывает большие трудности в связи с тем, что требует достоверного сведения о состоянии пластов и оклоскважинной зоны (с использованием аналитических методов) либо детального анализа логарифмической производной давления с участием квалифицированных интерпретаторов.

В этой связи перспективным направлением развития методов диагностики фильтрационных потоков горизонтальных скважин, оснащенных стационарными информационными измерительными телеметрическими системами, являются адаптивные технологии идентификации, позволяющие не только выделять фильтрационные потоки, но и определять параметры 
нефтяных пластов и время завершения гидродинамических исследований в процессе их проведения в промысловых условиях без участия квалифицированного интерпретатора [11-13].

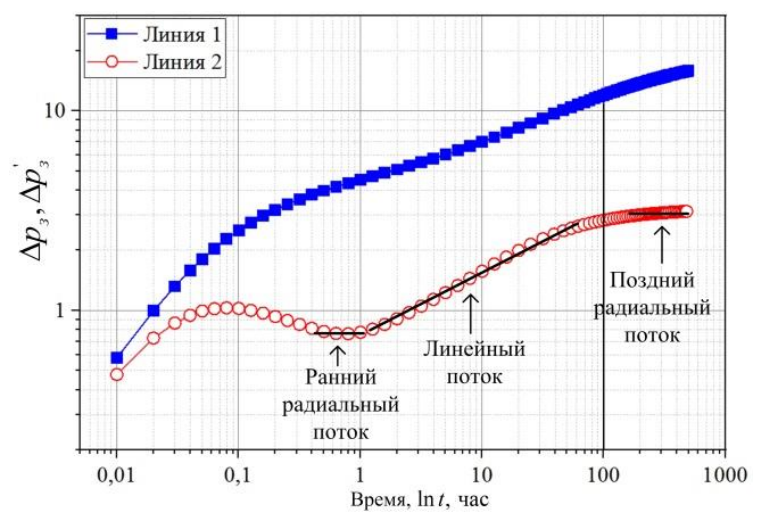

Рис. 1. Типовая кривая восстановления давления $\Delta p_{3}$ (линия 1) и ее производной $\Delta p_{3}^{\prime}$ (линия 2)

Fig. 1. Pressure buildup curve $\Delta p_{3}$ (line 1) and its derivative $\Delta p_{3}^{\prime}$ (line 2)

В данной работе, в развитие адаптивных технологий диагностики для выделения фильтрационных потоков, предлагается использовать зависящие от времени детерминированные моменты давлений (ДМД) $\mu_{k}(t)$ вида:

$$
\begin{gathered}
\mu_{k}(t)=\int_{t_{0}}^{t}\left(p_{\text {пл }}(t)-p_{3}^{*}(t)\right) t^{k} d t+ \\
+\int_{t}^{t_{p}}\left(p_{\text {пл }}(t)-p_{3}\left(t_{n}+\tau\right)\left(t_{n}+\tau\right)^{k} d \tau, k=0,1,2,\right.
\end{gathered}
$$

где пластовое $p_{\text {пл }}(t) \rightarrow p_{\text {пл }}\left(t_{p}\right), t \rightarrow t_{p}$ и забойное давление $p_{3}\left(t_{n}+\tau\right)$ на недовосстановленном участке КВД $\left(t, t_{p}\right]$ представлены неизвестными однозначными функциями текущего времени $t$ с момента остановки скважины $t_{0} ; t_{p}-$ время восстановления забойного давления до пластового; $p_{3}{ }^{*}(t)$ - забойное давление.

В частном случае, при $t=t_{\mathrm{K}}$, где $t_{\mathrm{K}}-$ время завершения гидродинамических исследований, ДМД (1) представляет собой основу известного традиционного метода определения типа и параметров нефтяных и газовых пластов $[14,15]$, проблемными моментами которого является определение момента времени завершения гидродинамических исследований $t_{\mathrm{\kappa}}$, пластового $p_{\text {пл }}\left(t_{p}\right)$ и забойного давлений $p_{3}\left(t_{n}+\tau\right)$ в процессе проведения испытаний. Для решения данной проблемы в [16] предложен адаптивный метод ДМД, позволяющий определять пластовое и забойное давление, а также время завершения гидродинамических исследований вертикальных скважин в процессе их проведения в промысловых условиях. Основой адаптивного метода ДМД являются оценки (1), которые предлагается использовать в данной работе для формирования диагностических критериев выделения фильтрационных режимов течения в процессе проведения испытаний горизонтальных скважин.

\section{Диагностические критерии выделения фильтрационных потоков}

В качестве диагностических параметров $d_{1}{ }^{*}(t), d_{2}{ }^{*}(t)$ для выделения приведенных на рис. 1 фильтрационных потоков в процессе проведения ГДИ горизонтальных скважин в работе использованы приближения:

$$
\begin{aligned}
d_{1}^{*}(t) & =\frac{\mathrm{d}\left(\mu_{1}^{*}(t) /\left(\mu_{0}^{*}(t)\right)^{2}\right)}{\mathrm{d} t}, \\
d_{2}^{*}(t) & =\mu_{0}^{*}(t) \mu_{2}^{*}(t) /\left(\mu_{1}^{*}(t)\right)^{2},
\end{aligned}
$$

где $\mu_{k}^{*}(t), k=0,1,2$ - оценки ДМД (1), в которых пластовые $p_{\text {пл }}^{*}(t)$ и забойные давления на недовосстановленном участке КВД $p_{3}^{*}(t+\tau)$ получены с использованием метода адаптивной идентификации $[11,17]$

$$
\begin{gathered}
p_{\text {пл }}^{*}(t)=f\left(t_{p}, \boldsymbol{\alpha}_{t}^{*}\left(\mathbf{h}_{t}^{*}\right)\right), \\
P_{3}^{*}(t+\tau)=f\left(t+\tau, \boldsymbol{\alpha}_{t}^{*}\left(\mathbf{h}_{t}^{*}\right)\right) .
\end{gathered}
$$

В (3), согласно методу адаптивной идентификации, оценки $\boldsymbol{\alpha}_{t}^{*}\left(\mathbf{h}_{t}^{*}\right)$ параметров модели забойного давления $f\left(t_{n}, \boldsymbol{\alpha}_{\mathbf{n}}\right)$ (6) и управляющих параметров $\mathbf{h}_{n}^{*}$ определяются из решения двух оптимизационных задач

$$
\begin{gathered}
\left.\boldsymbol{\alpha}_{n}^{*}\left(\mathbf{h}_{n}\right)=\arg \min _{\boldsymbol{\alpha}_{n}} \Phi\left(t_{n}, \boldsymbol{\alpha}_{n}, \mathbf{h}_{n}\right)\right), \\
\mathbf{h}_{n}^{*}=\arg \min _{\mathbf{h}_{n}} J_{0}\left(t_{n}, \boldsymbol{\alpha}_{n .}^{*}\left(\mathbf{h}_{n}\right)\right), n=\overline{1, n}_{\mathrm{\kappa}} .
\end{gathered}
$$

Здесь $\Phi\left(\boldsymbol{\alpha}_{n}, \boldsymbol{\beta}_{n}\right)=J_{0}\left(\boldsymbol{\alpha}_{n}, h_{3, n}\right)+J_{a}\left(\boldsymbol{\alpha}_{n}, h_{p, n}, h_{k, n}\right)-$ комбинированный показатель качества модели вида:

$$
\left\{\begin{array}{l}
p_{3}^{*}\left(t_{n}\right)=p_{3}\left(t_{0}\right)+f\left(t_{n}, \boldsymbol{\alpha}_{\mathbf{n}}\right)+\xi\left(t_{n}\right), n=\overline{1, n_{\mathrm{\kappa}}}, \\
h_{\mathrm{к}, n} \cdot \bar{p}_{\text {пл }}=p_{3}\left(t_{0}\right)+f\left(T, \boldsymbol{\alpha}_{\mathbf{n}}\right)+\eta_{n},
\end{array}\right.
$$

в которой $f\left(t_{n}, \boldsymbol{\alpha}_{n}\right)$ - феноменологическая модель забойного давления $p_{3}^{*}\left(t_{n}\right)$ [18]; $\boldsymbol{\alpha}_{\mathbf{n}}=\left(\alpha_{1}\left(t_{n}\right), \alpha_{2}\left(t_{n}\right), \ldots, \alpha_{m}\left(t_{n}\right)\right)-$ вектор параметров, представленный неизвестными однозначными функциями дискретного времени $t_{n}$; $\bar{p}_{\text {пл }}, T-$ экспертные оценки пластового давления и времени восстановления забойного давления до пластового;

$$
\begin{gathered}
J_{0}\left(t_{n}, \boldsymbol{\alpha}_{n}, h_{3, n}\right)= \\
=\sum_{i=1}^{n_{\mathrm{K}}} w_{n}\left(\frac{t_{n}-t_{i}}{h_{3, n}} \psi_{0}\left(\Delta p_{3}^{*}\left(t_{n}\right)-f_{0}\left(t_{n}, \boldsymbol{\alpha}_{n}\right)\right)-\right.
\end{gathered}
$$

показатель качества модели КВД $f\left(t_{n}, \boldsymbol{\alpha}_{\mathbf{n}}\right)$; $J_{a}\left(\boldsymbol{\alpha}_{n}, \mathbf{h}_{n}\right)=\psi_{a}\left(h_{\text {к, } n} \cdot \bar{p}_{\text {пл }}-\left(p_{3}\left(t_{0}\right)+f\left(T, \boldsymbol{\alpha}_{\mathbf{n}}\right)\right)\right)-$ показатель качества модели пластового давления; $\Delta p_{3}^{*}\left(t_{n}\right)=p_{3}^{*}\left(t_{n}\right)-p_{3}\left(t_{0}\right) ; \psi_{0}, \psi_{a}-$ известные функции [17]; $\mathbf{h}_{n}^{*}=\left(h_{3, n}^{*}, h_{p, n}^{*}, h_{\kappa . n}^{*}\right)-$ вектор оценок управляющих параметров (забывания $h_{3, n}^{*}$, регуляризации $h_{p . n}^{*}$, корректировки $h_{\text {к.n }}^{*}$ ); где 


$$
w_{n}\left(\left(t_{n}-t_{i}\right) / h_{3, n}\right), i=\overline{1, n}-
$$

значения весовой функции $w(x)$, введенной для организации процесса адаптивной идентификации,

$$
w(0)=1, w\left(x_{1}\right) \leq w\left(x_{2}\right) \text { при }\left|x_{1}\right|<\left|x_{2}\right| .
$$

Целесообразность использования оценок (2) для выделения фильтрационных потоков объясняется тем, что в области линейного и позднего радиального потоков диагностические параметры $d_{1}^{*}(t)$ и $d_{2}^{*}(t)$ имеют выраженные экстремумы (рис. 3, 4). В табл. 1 приведены критерии выделения фильтрационных потоков, основанные на диагностических параметрах (2).

Таблица 1. Диагностические критерии выделения фильтрачионных потоков

Table 1. Diagnostic criteria for identifying flow regimes

\begin{tabular}{|c|c|c|}
\hline $\begin{array}{c}\text { Фильтрационные } \\
\text { потоки } \\
\text { Flow regimes }\end{array}$ & $\begin{array}{c}\text { Киагностические } \\
\text { критерии } \\
\text { Diagnostic criteria }\end{array}$ & $\begin{array}{c}\text { Критерии моментов } \\
\text { времени потоков } \\
\text { Criteria for estimating } \\
\text { time of regimes }\end{array}$ \\
\hline $\begin{array}{c}\text { Ранний } \\
\text { радиальный } \\
\text { Early-radial }\end{array}$ & $\min _{t}\left(d_{1}^{*}(t)-d_{2}^{*}(t)\right)$ & $t_{\mathrm{pp}}=\underset{t}{\operatorname{mpg} \min _{t}\left(d_{1}^{*}(t)-d_{2}^{*}(t)\right)}$ \\
\hline $\begin{array}{c}\text { Линейный } \\
\text { Linear }\end{array}$ & $\min _{t}\left(d_{1}^{*}(t)\right)$ & $t_{\text {Л }}=\underset{t}{\arg \min _{t}\left(d_{1}^{*}(t)\right)}$ \\
\hline $\begin{array}{c}\text { Поздний } \\
\text { радиальный } \\
\text { Late-radial }\end{array}$ & $\max _{t}\left(d_{2}(t)\right)$ & $t_{\text {пр }}=\arg \max _{t}\left(d_{2}(t)\right)$ \\
\hline
\end{tabular}

Результаты диагностики фильтрационных режимов течения по промысловым испытаниям скважин

Результаты изучения моделей и алгоритмов метода адаптивной диагностики фильтрационных режимов течения по данным нестационарных испытаний нефтяных горизонтальных скважин № 1 и 2 месторождения Тюменской области представлены на рис. 3, 4 и в табл. 3. Исходные и дополнительные данные скважин приведены в табл. 2. На рис. 2 изображены промысловые значения забойных давлений $p_{3}^{*}\left(t_{i}\right)$, $i=\overline{1, n}(2)$, полученные в процессе проведения ГдИ скважин, в полулогарифмических координатах $\left(p_{3}^{*}(t)\right.$-по оси $Y, \ln t-$ по оси $\left.X\right)$.

На рис. 3, 4 (линией 1) изображены сглаженные значения логарифмической производной $y(t)=\lg \left(\mathrm{d} p_{3}^{*}(t) / \mathrm{d}(\ln t)\right)$ забойного давления $p_{3}^{*}(t)$, используемые в графоаналитических методах для выделения фильтрационных потоков [19-24]. Оценки диагностических параметров (2) с использованием в модели (6) логистической феноменологической функции Хоанг-Ан с переменными, зависящими от времени параметрами $b(t), \alpha_{1}(t), \alpha_{2}(t)$ [18]

$$
\begin{gathered}
f\left(t, b(t), \alpha_{1}(t), \alpha_{2}(t)\right)= \\
=\left(1-e^{-b(t) \cdot t}\right)^{-1} \cdot\left(\alpha_{1}(t)+\alpha_{2}(t) \cdot \ln t\right),
\end{gathered}
$$

изображены на рис. 3, 4 линиями 2, 3 .

Следует отметить, что для модели (6) (с учетом (7)), представленной в матричном виде

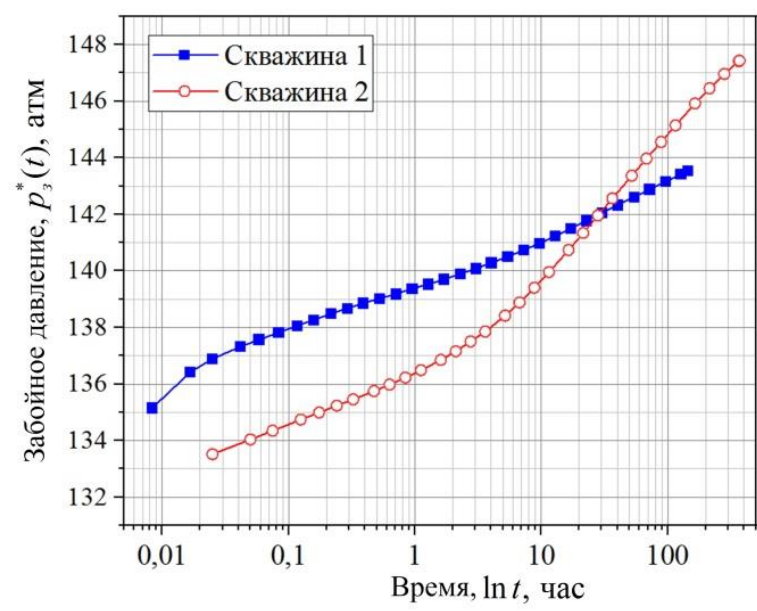

Pис. 2. Кривые восстановления давления горизонтальных скважин № 1 и 2 в полулогарифмических координатах

Fig. 2. Build-up curves of horizontal wells no. 1 and 2 in semi-log plot

Таблица 2. Исходнье и дополнительные априорные

\begin{tabular}{|c|c|c|}
\hline \multirow{2}{*}{$\begin{array}{l}\text { Исходные и дополнительные данные } \\
\text { Initial and additional a prior data }\end{array}$} & \multicolumn{2}{|c|}{ Скважины/Wells } \\
\hline & № 1 & № 2 \\
\hline $\begin{array}{l}\text { Радиус скважины } r_{\mathrm{c}}, \mathrm{M} \\
\text { Well radius } r_{\mathrm{c}}, \mathrm{m}\end{array}$ & 0,108 & 0,108 \\
\hline \begin{tabular}{|l|} 
Пористость $\phi$ \\
Porosity $\phi$ \\
\end{tabular} & 0,13 & 0,13 \\
\hline \begin{tabular}{|l|} 
Толщина пласта $h, \mathrm{~m}$ \\
Formation thickness $h, \mathrm{~m}$
\end{tabular} & 5,4 & 7,2 \\
\hline $\begin{array}{l}\text { Динамическая вязкость нефти } \mu, \text { сП } \\
\text { Oil dynamic viscosity } \mu, \mathrm{cP}\end{array}$ & 3,92 & 3,92 \\
\hline $\begin{array}{l}\text { Температура при стандартном условии } T_{\text {ск }} \\
\left(+20^{\circ} \mathrm{C}\right) \mathrm{K} \\
\text { Temperature, standard conditions } T_{\text {ск }}, \\
\left(+20^{\circ} \mathrm{C}\right) \mathrm{K}\end{array}$ & 293 & 293 \\
\hline $\begin{array}{l}\text { Пластовая температура } T_{\text {пл }}, \mathrm{K} \\
\text { Reservoir temperature } T_{\text {пл }}, \mathrm{K} \\
\end{array}$ & 298 & 298 \\
\hline $\begin{array}{l}\text { Сжимаемость пласта } c_{t}, \text { aтм }^{-1} \\
\text { Reservoir compressibility } c_{t}, \mathrm{~atm}^{-1}\end{array}$ & $4,72 \cdot 10^{-4}$ & $4,72 \cdot 10^{-4}$ \\
\hline $\begin{array}{l}\text { Стандартное давление } P_{\text {ст }} \text {, атм } \\
\text { Standard pressure } P_{\text {ст }} \text {, atm } \\
\end{array}$ & 1,033 & 1,033 \\
\hline $\begin{array}{l}\text { Дебит скважины до остановки } q_{0}^{*}, \mathrm{~m}^{3} / \text { сут } \\
\text { Well rate before shut-in } q_{0}^{*}, \mathrm{~m}^{3} / \text { day }\end{array}$ & 137 & 163,2 \\
\hline $\begin{array}{l}\text { Экспертная оценка пластового } \\
\text { давления, атм } \\
\text { Expert estimate of reservoir pressure, atm }\end{array}$ & 142 & 150 \\
\hline $\begin{array}{l}\text { Экспертная оценка времени начала } \\
\text { линейного потока, } \bar{t}_{\mathrm{л}}, \mathrm{ч} \\
\text { Expert estimate of linear regime beginning } \\
\bar{t}_{\text {л }}, \text { hour }\end{array}$ & 2 & 2 \\
\hline
\end{tabular}
данные горизонтальных скважин

Table 2. Initial and additional a priori data of horizontal wells

$$
\left\{\begin{array}{l}
\Delta \mathbf{p}_{3, n}^{*}=F_{n}\left(b_{n}\right) \boldsymbol{\alpha}_{n}+\xi_{n}, \\
H_{\kappa, n} \overline{\boldsymbol{\alpha}}=f_{a}\left(\boldsymbol{\alpha}_{n}\right)+\eta_{n}, n=\overline{1, n_{\mathrm{\kappa}}},
\end{array}\right.
$$

задача параметрической идентификации (4), (5) при использовании частных квадратичных показателей качества $\psi_{0}(x)=\psi_{a}(x)=x^{2}$ сводится к решению двух одномерных оптимизационных задач: 


$$
\begin{gathered}
b_{n}^{*}\left(h_{\mathrm{\kappa}, n}\right)= \\
=\arg \min _{b_{n}}\left(\left\|\Delta \mathbf{p}_{3, n}^{*}-F_{n}\left(b_{n}\right) \boldsymbol{\alpha}_{n}^{*}\left(b_{n}, h_{\mathrm{\kappa}, n}^{*}\right)\right\|_{W\left(h_{3, n}\right)}^{2}\right), \\
h_{\mathrm{k}, n}^{*}= \\
=\arg \min _{h_{\mathrm{k}, n}}\left(\left\|\Delta \mathbf{p}_{3, n}^{*}-f\left(b_{n}\right) F_{n} \boldsymbol{\alpha}_{n}^{*}\left(b_{n}, h_{\mathrm{\kappa}, n}\right)\right\|_{W\left(h_{3, n}\right)}^{2}\right),
\end{gathered}
$$

где оценки $\boldsymbol{\alpha}_{n}^{*}$ параметра $\boldsymbol{\alpha}_{n}$ модели (8) определяются из решения системы линейных алгебраических уравнений вида

$$
\begin{aligned}
& \left(F_{n}\left(b_{n}\right)^{T} K\left(h_{3, n}\right) F_{n}\left(b_{n}\right)+h_{\mathrm{p}, n} I\right) \boldsymbol{\alpha}_{n}= \\
& =F_{n}\left(b_{n}\right)^{T} K\left(h_{3, n}\right) \Delta \mathbf{p}_{3, n}^{*}+h_{\mathrm{p}, n} H_{\mathrm{\kappa}, n} \overline{\boldsymbol{\alpha}},
\end{aligned}
$$

$\Delta \mathbf{p}_{3, n}^{*}=\left(p_{3, n}^{*}-p_{3}^{*}\left(t_{0}\right), n=\overline{n_{\text {нл }}, n_{\text {к }}}\right)-$ вектор значений разности забойного давления;

$$
F_{n n}^{T}\left(b_{n}\right)=\left(\begin{array}{cccc}
y_{1}\left(b_{1}\right) & y_{2}\left(b_{2}\right) & \ldots & y_{n}\left(b_{n}\right) \\
y_{1}\left(b_{1}\right) x_{1} & y_{2}\left(b_{2}\right) x_{2} & \ldots & y_{n}\left(b_{n}\right) x_{n}
\end{array}\right)-
$$

матрица, в которой

$$
\begin{gathered}
y_{i}\left(b_{i}\right)=\left(1-e^{-b(i) \cdot i}\right)^{-1}, x_{i}=\lg \left(t_{i}\right), \quad i=\overline{1, n_{\mathrm{r}}} ; \\
K\left(h_{3, n}\right)=\operatorname{diag}\left(w\left(\left(t_{n}-t_{n-i}\right) / h_{3, n}\right), i=\overline{0, n-1}\right)-
\end{gathered}
$$

диагональная матрица весовых функций $w\left(\left(t_{n}-t_{n-i}\right) / h_{3, n}\right)$ с параметром забывания $h_{3, n} ; h_{\mathrm{p}, n}$ - параметр регуляризации; $H_{\mathrm{\kappa}, n}=\operatorname{diag}\left(0, h_{\mathrm{\kappa}, n}\right)$ - диагональная матрица; $\Gamma=\operatorname{diag}(0,1) ; T-$ символ транспонирования; $\xi_{n}, \eta_{n}-$ случайные величин.

Оптимизационные задачи (9), (10) решаются с применением метода дихотомии [25]. Оценки параметров $b_{n}^{*}, h_{\mathrm{r}, n}^{*}, \boldsymbol{\alpha}_{n},(9)-(11)$, получены при постоянных единичных значениях весовой функции $w\left(\left(t_{n}-t_{n-i}\right) / h_{3, n}\right)$ в текущем интервале обработки $\left[t_{n}-t_{n-4}\right]$, содержащем пять значений забойного давления $\left(h_{3, n}=5\right),\left(w_{n}\left(\left(t_{n}-t_{n-i}\right) / h_{3, n}\right)=1\right.$ при $\left(t_{n}-t_{n-i}\right) \leq h_{3, n}=t_{n}-t_{n-4}$ и $w_{n}\left(\left(t_{n}-t_{n-i}\right) / h_{3, n}\right)=0$ при $\left(t_{n}-t_{n-i}\right)>h_{3, n}$, $i=\overline{0, n-1}), h_{p, n}=h_{p}=0,01$.

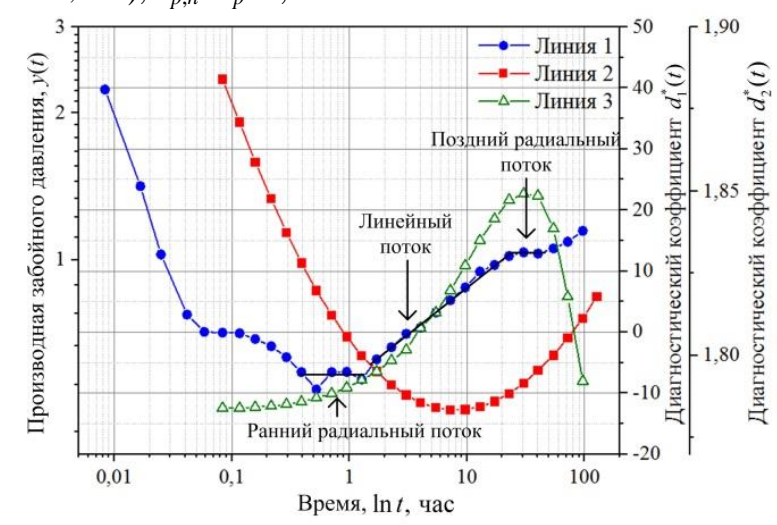

Pис. 3. Диагностические графики скважинь № 1 (линия 1 - производная забойного давления $y(t)$, линии 2, 3 - диагностические коэффициенты $\left.d_{1}^{*}(t), d_{2}^{*}(t)(2)\right)$

Fig. 3. Diagnostic plot of well no. 1 (line 1 -pressure derivative, line $2,3-$ diagnostic coefficients $\left.d_{1}^{*}(t), d_{2}^{*}(t)(2)\right)$

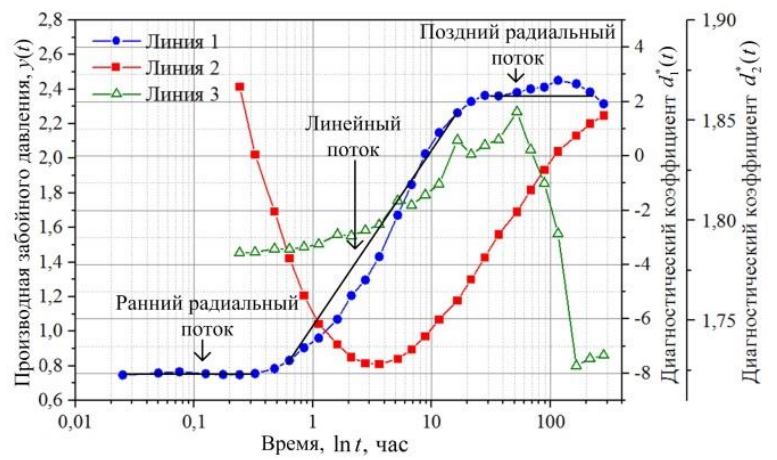

Pис. 4. Диагностические графики скважсины № 2 (линия 1 - производная забойного давления $y(t)$, линии 2, 3 - диагностические коэффициенты

\begin{tabular}{|c|c|c|c|c|}
\hline 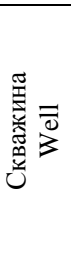 & $\begin{array}{l}\text { Метод диагно- } \\
\text { стики потоков } \\
\text { Flow diagnostic } \\
\text { method }\end{array}$ & $\begin{array}{c}\text { Время } \\
\text { завершения } \\
\text { раннего } \\
\text { радиально- } \\
\text { го потока } \\
\text { Early-radial } \\
\text { flow end } \\
\text { time }\end{array}$ & $\begin{array}{c}\text { Время } \\
\text { централь- } \\
\text { ной части } \\
\text { линейного } \\
\text { потока } \\
\text { Middle time } \\
\text { of the linear } \\
\text { flow }\end{array}$ & $\begin{array}{c}\text { Время } \\
\text { начала } \\
\text { позднего } \\
\text { радиально- } \\
\text { го потока } \\
\text { Beginning } \\
\text { of late- } \\
\text { radial flow }\end{array}$ \\
\hline & $\begin{array}{l}\text { Графоаналити- } \\
\text { ческий } \\
\text { Graphoanalytical }\end{array}$ & 1,05 & 7,1 & 13,8 \\
\hline № 1 & $\begin{array}{l}\text { Адаптивный с } \\
\text { идентификацией } \\
\text { ДП } \\
\text { Adaptive method } \\
\text { with identification } \\
\text { of diagnostic } \\
\text { parameter }\end{array}$ & 1,08 & 7,4 & 13,1 \\
\hline & $\begin{array}{l}\text { Графоаналити- } \\
\text { ческий } \\
\text { Graphoanalytical }\end{array}$ & 0,35 & 1,5 & 14 \\
\hline № 2 & $\begin{array}{l}\text { Адаптивный с } \\
\text { идентификацией } \\
\text { ДП } \\
\text { Adaptive method } \\
\text { with identification } \\
\text { of diagnostic } \\
\text { parameter }\end{array}$ & 0,41 & 1,43 & 14,2 \\
\hline
\end{tabular}
$\left.d_{1}^{*}(t), d_{2}^{*}(t)(2)\right)$

Fig. 4. Diagnostic plot of well no. 2 (line 1 - pressure derivative, line 2,3 - diagnostic coefficients $\left.d_{1}^{*}(t), d_{2}^{*}(t)(2)\right)$

Таблица 3. Результаты диагностики фильтращионных потоков скважсин № 1 и 2

Table 3. Results of flow regimes diagnostic of wells no. 1 and 2

Из рис. 3, 4 и табл. 3 видно, что полученные с использованием приведенных в табл. 1 диагностических критериев моменты времени фильтрационных потоков не уступают по точности моментам времени, определенным с использованием графоаналитического метода диагностики потоков по производной забойного давления. Например, для скважины № 1 момент времени завершения раннего радиального потока, полученного адаптивным методом, практически совпадает с моментом времени, полученным на основе графоаналитического метода, и составляет 1,05 часа после остановки скважины. 


\section{Заключение}

Предложены диагностические критерии детерминированных моментов давлений для выделения фильтрационных потоков в процессе гидродинамических исследований горизонтальных скважин нефтяных месторождений.

По результатам исследований двух скважин нефтяного месторождения показано, что разработан-

\section{СПИСОК ЛИТЕРАТУРЫ}

1. Развитие геофизического и гидродинамического мониторинга на этапе перехода к разработке объектов с трудноизвлекаемыми запасами нефти / В.Г. Мартынов, А.И. Ипатов, М.И. Кременецкий, Д.Н. Гуляев, В.М. Кричевский, В.В. Кокурина, С.И. Мельников // Нефтяное хозяйство. - 2014. - № 3. С. 106-109.

2. Кременецкий М.И., Ипатов А.И., Гуляев Д.Н. Информационное обеспечение и технологии гидродинамического моделирования нефтяных и газовых скважин. - М.; Ижевск: Институт компьютерных исследований, 2012. - 896 с.

3. Joshi S.D. Horizontal well technology. - Oklahoma: PenWell publ comp., 1991. - 381 p.

4. Bourdet D., Ayoub J.A., Pirard Y.M. Use of pressure derivative in well test interpretation // Society of Petroleum Engineers. 1984. - № 12777. - P. 293-302.

5. Хисамов Р.С., Сулейманов Э.И., Фархуллин Р.Г. Гидродинамические исследования скважин и методы обработки результатов измерений. - М.: ОАО «ВНИИОЭНГ», 2000. - 228 с.

6. Kuchuk F.J. Well testing and interpretation for horizontal wells // Journal of Petroleum Technology. - 1995. - V. 47. - № 1. P. 36-41.

7. Odeh A.S. Transient flow behavior of horizontal well: Pressure drawdown and buildup analysis of horizontal wells in anisotropic media // Society of Petroleum Engineers. - 1987. - № 14250. P. 683-697.

8. Kolin S.K., Kureija T., Grebenar D. Pressure build-up test analysis of the hydrocarbon reservoir system with the multiphase flow // The mining-geology-petroleum engineering bulletin. - 2018. № 9. - P. 1-15.

9. Kamal M.M. Transient well testing. - Texas: Society of Petroleum Engineers, 2009. - 863 p.

10. Иктисанов В.А. Совершенствование методик интерпретации кривых восстановления давления горизонтальных скважин // Нефтяное хозяйство. - 2002. - № 2. - С. 56-59.

11. Сергеев В.Л., Ву К.Д. К оптимизации адаптивных алгоритмов идентификации и интерпретации гидродинамических исследований с учетом влияния ствола скважины // Доклады ТУСУР. - 2016. - Т. 19. - № 3. - С. 98-102.

12. Романова Е.В., Сергеев В.Л. Адаптивная идентификация кривой восстановления давления горизонтальных скважин с диагностикой потоков // Известия Томского политехнического университета. - 2013. - Т. 323. - № 5. - С. 20-25. ные диагностические критерии практически не уступают по точности графоаналитическому методу диагностики потоков, основанному на визуальном анализе производной забойного давления, и позволяют выделять фильтрационные потоки в процессе проведения нестационарных испытаний скважин по кривым восстановления забойного давления без участия квалифицированного интерпретатора.

13. Сергеев В.Л., Донг Ван Хоанг, Хагай Д.Э. Модели и алгоритмы адаптивного метода диагностики фильтрационных потоков в процессе испытаний горизонтальных скважин // Доклады ТУСУР. - 2019. - Т. 22. - № 2. - С. 90-95.

14. Басович И.Б., Капцанов Б.С. Выбор фильтрационных моделей по данным гидродинамических исследований скважин // Нефтяное хозяйство. - 1980. - № 3. - С. 44-47.

15. Булгаков С.А., Ольховская Б.А. Повышение информативности исследования нефтяных скважин на основе метода ДМД // Геология, геофизика и разработка нефтяных и газовых месторождений. - 2011. - № 1. - С. 54-57.

16. Сергеев В.Л., Нгуен Т.Х.Н., Ву К.Д. Модели и алгоритмы идентификации начального участка забойного давления в адаптивном методе детерминированных моментов давлений // Доклады ТУСУР. - 2018. - Т. 21. - № 4. - С. 105-115.

17. Сергеев В.Л. Интегрированные системы идентификации. Томск: Изд-во Том. политехн. ун-та, 2011. - 198 с.

18. Сергеев В.Л., Донг Ван Хоанг, Фам Динь Ан. Адаптивная идентификация гидродинамических исследований горизонтальных скважин на прогнозирующих моделях // Известия Томского политехнического университета. Инжиниринг георесурсов. - 2019. - Т. 330. - № 1. - С. 165-172.

19. Bourdet D. Well test analysis: the use of advanced interpretation models. - Amsterdam: Elsevier Science, 2002. - 426 p.

20. Engler T., Tiab D. Analysis of pressure and pressure derivative without type-curve matching. Horizontal well tests in anisotropic media // Journal of Petroleum Science and Engineering. - 1996. № 15. - P. 153-168.

21. Ozkan E., Raghavan R., Joshi S.D. Horizontal-well pressure analysis // Society of Petroleum Engineers. - 2007. - № 16378. P. 567-575.

22. Lu J., Cunha L.B., Lu T. New solution for well test analysis of horizontal wells // Society of Petroleum Engineers. - 2002. № 78972. - P. 1-7.

23. Pressure and pressure derivative interpretation for horizontal wells in compressible formations / F.H. Escobar, Yu-Long Zhao, C. Urazan, C.M. Trujullo // Journal of Geophysics and Engineering. -2018 . - № 15. - P. 1551-1560.

24. Kuchuk F.J., Onur M., Hollaender F. Pressure transient formation and well testing: convolution, deconvolution and nonline estimation. - Amsterdam: Elsevier Science, 2010. -389 p.

25. Пантелеев А.В., Летова Т.А. Методы оптимизации в примерах и задачах. - СПб.: Изд-во «Лань», 2015. - 512 с.

Поступила: 01.11.2019 г.

\section{Информация об авторах}

Сергеев В.Л., доктор технических наук, профессор отделения нефтегазового дела Инженерной школы природных ресурсов Национального исследовательского Томского политехнического университета.

Донг Ван Хоанг, аспирант отделения нефтегазового дела Инженерной школы природных ресурсов Национального исследовательского Томского политехнического университета.

Хагай Д.Э., руководитель проекта, ООО «Альтаир».

Игнатенко А.В., директор ТОО «АЛСТРОНТЕЛЕКОМ». 
UDC 519.688:622.276

\title{
DIAGNOSTIC CRITERIA FOR IDENTIFICATION OF FILTRATION FLOWS DURING HORIZONTAL WELL TESTS
}

\author{
Viktor L. Sergeev', \\ SVL00@tpu.ru
}

\section{Dong Van Hoang',} hoang.tpu@gmail.com

Danil E. Khagay², hagayd@mail.ru

\author{
Aleksandr V. Ignatenko3, \\ IgnatenkoAV@alstron.kz \\ ${ }^{1}$ National Research Tomsk Polytechnic University, \\ 30, Lenin avenue, Tomsk, 634050, Russia. \\ 2 Altair company, \\ 71D, IrkutskH street, Tomsk, 634062, Russia. \\ 3 LLP «Alstrontelecom», \\ 42, Timiryazeva street, Almaty, 050032, Kazakhstan.
}

The relevance of the research is caused by the need to identify the filtration flow regimes for operational determination of hydrodynamic parameters of oil reservoirs during horizontal well tests by bottomhole pressure buildup curves

The main aim of the research is to develop diagnostic criteria of deterministic pressure moments for identification of filtration flow regimes during horizontal well tests in oil fields by pressure recovery curve.

The methods are based on theory and practice of non-stationary horizontal well tests, linear algebra, system identification, system analysis. The proposed diagnostic criteria were tested on the basis of field data of horizontal well tests in oil field by bottomhole pressure buildup curve.

The results. The authors have developed the diagnostic criteria for identification of early-radial, linear and late-radial (pseudoradial) flow regimes on pressure buildup curve during horizontal well tests in the oil fields.

The diagnostic criteria are based on the estimates of deterministic pressure moments obtained during horizontal well tests. To define the unknown values of reservoir and bottomhole pressures the adaptive identification algorithms and phenomenological forecasting models of bottomhole pressure with variable parameters were used taking into account additional a priori information about reservoir pressure. The processing of test results of two horizontal wells in an oil field was carried out, it is shown that the proposed diagnostic criteria are not inferior in accuracy to the graph-analytical method for identifying filtration regimes and allow determining the time of the beginning and completion of the filtration flows, as well as the time of well shut-in during the tests without the participation of a qualified interpreter.

\section{Key words:}

Diagnostic criteria, filtration flows, adaptation, horizontal wells, well hydrodynamic study,

identification, pressure buildup curve, oil reservoirs.

\section{REFERENCES}

1. Martynov V.G., Ipatov A.I., Kremenetskiy M.I., Gulyaev D.N., Krichevskiy V.M., Kokurina V.V., Melnikov S.I. Permanent reservoir monitoring by logging gages at the stage of tight oil recovery. Neftyanoe khozyaystvo, 2014, no. 1, pp. 106-109. In Rus.

2. Kremenetsky M.I., Ipatov A.I., Gulyaev D.N. Informationoe obespechenie i tekhnologii gidrodinamicheskogo modelorovaniya neftyanykh i gazovykh zalezhey [Information and technology of hydrodynamic modeling of oil and gas deposits]. Moscow; Izhevsk, Institute of computer research Press, 2012.896 p.

3. Joshi S.D. Horizontal well technology. Oklahoma, PenWell publ. comp., 1991. $381 \mathrm{p}$.

4. Bourdet D., Ayoub J.A., Pirard Y.M. Use of pressure derivative in well test interpretation. Society of Petroleum Engineers, 1984, no. 12777 , pp. $293-302$.

5. Khisamov R.S., Suleymanov E.I., Farkhullin R.G. Gidrodinamich eskie issledovaniya skvazhin i metody obrabotki rezultatov izmereny [Hydrodynamic studies of wells and methods of processing the results of measurements]. Moscow, VNIIOENG Publ., 2000. $228 \mathrm{p}$.

6. Kuchuk F.J. Well testing and interpretation for horizontal wells. Journal of Petroleum Technology, January 1995, vol. 47, no. 1, pp. 36-41.
7. Odeh A.S. Transient flow behavior of horizontal well: Pressure drawdown and buildup analysis of horizontal wells in anisotropic media. Society of Petroleum Engineers, 1987, no. 14250, pp. 683-697.

8. Kolin S.K., Kureija T., Grebenar D. Pressure build-up test analysis of the hydrocarbon reservoir system with the multiphase flow. The mining-geology-petroleum engineering bulletin, 2018, no. 9, pp. 1-15.

9. Kamal M.M. Transient well testing. Texas, Society of Petroleum Engineers, 2009. $836 \mathrm{p}$.

10. Iktisanov V.A. Sovershenstvovanie metodik interpretatsii krivykh vosstanovleniya davleniya gorizontalnykh skvazhin [Improving the interpretation techniques of buildup curves of horizontal wells]. Neftyanoe khozyaystvo, 2002, no. 2, pp. 56-59. In Rus.

11. Sergeev V.L., Vu Quang Duc. Optimization of adaptive algorithms for identification and interpretation of hydrodynamics research considering wellbore storage. Proceeding of TUSUR University, 2016, no. 3 (19), pp. 98-102. In Rus.

12. Romanova E.V., Sergeev V.L. Adaptive interpretation of pressure recovery curve of horizontal wells with flow diagnostics. Bulletin of the Tomsk Polytechnic University, 2013, vol. 323, no. 5, pp. 20-25. In Rus. 
13. Sergeev V.L., Dong V.H., Khagay D.E. Models and algorithms of adaptive method to detect filtration flow during horizontal well testing. Proceeding of TUSUR University, 2019, no. 2 (22), pp. 90-95. In Rus.

14. Basovich I.B., Kartsanov B.S., Vybor filtrationnykh modeley po dannym gidrodinamicheskikh issledovaniy [Selection of filtration models based on well testing data]. Neftyanoe khozyaystvo, 1980, no. 3, pp. 44-47.

15. Bugalkov S.A., Olkhovskaya B.A. Povyshenie informativnosti issledovaniya neftyanykh skvazhin na osvove medofa DMD [Improving the informativeness of wells test based on the DMD method]. Geologiya, geofizika i razrabotka neftyanykh i gazovykh mestorozhdeniy, 2011, no. 1, pp. 54-57.

16. Sergeev V.L., Nguyen T.H.P., Vu Q.D. Models and algorithms for identification of oil and gas reservoirs by the adaptive method of deterministic pressure moments. Proceeding of TUSUR University, 2018, no. 4 (21), pp. 109-115. In Rus.

17. Sergeev V.L. Integrirovannye sistemy identifikatsii [Integrated identification system]. Tomsk, Tomsk Polytechnic University Publ. house, 2011. $198 \mathrm{p}$.

18. Sergeev V.L., Dong Van Hoang, Pham Dinh An. Adaptive interpretation of the results of horizontal well production testing using forecasting models. Bulletin of the Tomsk Polytechnic University. Geo Assets Engineering, 2019, vol. 330, no. 1, pp. 165-172. In Rus.

Information about the authors
19. Bourdet D. Well test analysis: the use of advanced interpretation models. Amsterdam, Elsevier Science, 2002. $426 \mathrm{p}$.

20. Engler T., Tiab D. Analysis of pressure and pressure derivative without type-curve matching. Horizontal well tests in anisotropic media. Journal of Petroleum Science and Engineering, 1996, no. 15 , pp. $153-168$.

21. Ozkan E., Raghavan R., Joshi S.D. Horizontal-well pressure analysis. Society of Petroleum Engineers, 2007, no. 16378, pp. 567-575.

22. Lu J., Cunha L.B., Lu T. New solution for well test analysis of horizontal wells. Society of Petroleum Engineers, 2002, no. 78972, pp. 1-7.

23. Escobar F.H., Yu-Long Zhao, Urazan C., Trujullo C.M. Pressure and pressure derivative interpretation for horizontal wells in compressible formations. Journal of Geophysics and Engineering, 2018, no. 15, pp. 1551-1560.

24. Kuchuk F.J., Onur M., Hollaender F. Pressure transient formation and well testing: Convolution, Deconvolution and Nonline Estimation. Amsterdam, Elsevier Science, 2010, 389 p.

25. Panteleev A.V., Letova T.A. Metody optimizatsii v primerakh $i$ zadachakh [Optimization methods in examples and tasks]. StPetersburg, Lan Publ., 2015. 512 p.

Received: 1 November 2019.

Viktor L. Sergeev, Dr. Sc., professor, National Research Tomsk Polytechnic University.

Dong Van Hoang, postgraduate student, National Research Tomsk Polytechnic University.

Danil E. Khagay, project manager, Altair company.

Aleksandr V. Ignatenko, director, LLP «Alstrontelecom». 